\title{
Author Index Volume 37 (2013)
}

The issue number is given in front of the pagination

Alafuzoff, I., see Natunen, T. (1) 217-232

Alafuzoff, I., see Popova, S.N. (3) 603-610

Alaniz, M.E., see Beharry, C. (3) 539-550

Alaylığlu, M., see Gezen-Ak, D. (1) 185-195

Anfossi, M., see Bernardi, L. (2) 285-289

Annweiler, C., M. Montero-Odasso, D.J. Llewellyn, S. Richard-Devantoy, G. Duque and O. Beauchet, Meta-Analysis of Memory and Executive Dysfunctions in Relation to Vitamin D (1) 147171

Antoni, G., see Magnusson, K. (1) 29-40

Antuono, P., see Smith, J.C. (1) 197-215

Anziano, R., see Ito, K. (1) 173-183

Araz, Ö.S., see Gezen-Ak, D. (1) 185-195

Ardis, T., see O’Hare, E. (1) 77-88

Arese, M., see Krako, N. (4) 747-758

Arosio, B., see Di Francesco, A. (1) 3-8

Asai, K., see Noguchi-Shinohara, M. (4) 691-698

Asif, A.R., see Gawinecka, J. (1) 51-61

Atasoy, İ.L., see Gezen-Ak, D. (1) 185-195

Avila, J., see Hernández, F. (3) 507-513

Bakchine, S., see Chaunu, M.-P. (4) 769-776

Balschun, D., see Lo, A.C. (1) 109-125

Barcikowska, M., see Mroczko, B. (2) 273-283

Barroso, C., see Pires, C. (2) 335-342

Bartzokis, G. see Raven, E.P. (1) 127-136

Başar, E., see Yener, G.G. (4) 759-767

Basurto-Islas, G., see Iqbal, K. (3) 469-474

Bayer, T.A., see Yang, H. (2) 297-308

Beauchet, O., see Annweiler, C. (1) 147-171

Beharry, C., M.E. Alaniz and A. del Carmen Alonso, Expression of Alzheimer-Like Pathological Human Tau Induces a Behavioral Motor and Olfactory Learning Deficit in Drosophila melanogaster (3) 539-550

Bennett, R., see Huber, B.R. (2) 309-323

Benzinger, T.L.S., see Gordon, B.A. (1) 41-50

Ber, I.L., see Chaunu, M.-P. (4) 769-776

Bereczki, E., see Wang, S. (3) 495-505

Berger, E., see Chamard, L. (4) 789-793

Bernardi, L., M. Gallo, M. Anfossi, M.E. Conidi, R. Colao, G. Puccio, S.A.M. Curcio, F. Frangipane,
A. Clodomiro, M. Mirabelli, F. Vasso, N. Smirne, R. Di Lorenzo, R. Maletta and A.C. Bruni, Role of TOMM40 rs 10524523 Polymorphism in Onset of Alzheimer's Disease Caused by the PSEN1 M146L Mutation (2) 285-289

Bertram, L., see Natunen, T. (1) 217-232

Berzuini, C., see Fox, M. (4) 809-821

Besser, L.M., see Qiu, W.Q. (2) 421-428

Bilgiç, B., see Gezen-Ak, D. (1) 185-195

Binder, L.I., see Ward, S.M. (3) 593-602

Blanchard, J., see Iqbal, K. (3) 469-474

Blazey, T., see Gordon, B.A. (1) 41-50

Blum, D., see Lo, A.C. (1) 109-125

Blum, D., see Van der Jeugd, A. (4) 777-788

Bolognin, S., see Iqbal, K. (3) 469-474

Bredesen, D.E., see Descamps, O. (2) 343-355

Brice, A., see Chaunu, M.-P. (4) 769-776

Brickman, A.M., see Mez, J. (1) 137-146

Brinton, R.D., see Zhao, L. (2) 403-419

Broersen, L.M., see Wiesmann, M. (1) 233-245

Browne, T.C., see Kelly, R.J. (1) 63-75

Bruni, A.C., see Bernardi, L. (2) 285-289

Bucossi, S., R. Polimanti, M. Ventriglia, S. Mariani, M. Siotto, F. Ursini, L. Trotta, F. Scrascia, A. Callea, F. Vernieri and R. Squitti, Intronic rs2147363 Variant in $A T P 7 B$ Transcription Factor-Binding Site Associated with Alzheimer's Disease (2) 453-459

Budson, A.E., see Qiu, W.Q. (2) 421-428

Buée, L., see Chaunu, M.-P. (4) 769-776

Buée, L., see Lo, A.C. (1) 109-125

Buée, L., see Van der Jeugd, A. (4) 777-788

Buée-Scherrer, V., see Chaunu, M.-P. (4) 769-776

Butts, A.M., see Smith, J.C. (1) 197-215

Callaerts-Vegh, Z., see Lo, A.C. (1) 109-125

Callea, A., see Bucossi, S. (2) 453-459

Caltagirone, C., see Perri, R. (1) 99-107

Calvo-Garrido, J., see Zheng, L. (4) 713-733

Campagna, J., see Descamps, O. (2) 343-355

Cao, H., see Cui, L. (1) 19-28

Cao, M., see Qian, W. (3) 529-538

Cao, Z., see Robinson, R.A.S. (4) 661-666 
Caracciolo, B., see Handels, R.L.H. (2) 357-365

Cardone, F., see Gawinecka, J. (1) 51-61

Carimalo, J., see Gawinecka, J. (1) 51-61

Carlesimo, G.A., see Perri, R. (1) 99-107

Carvajal, F.J., see Zolezzi, J.M. (4) 735-746

Cattaneo, A., see Krako, N. (4) 747-758

Cedazo-Minguez, A., see Zheng, L. (4) 713-733

Cente, M., see Kazmerova, Z. (2) 251-272

Cernak, I., see Huber, B.R. (2) 309-323

Chai, G.-S., see Duan, D.-X. (4) 795-808

Chamard, L., D. Wallon, A. Pijoff, E. Berger, G. Viennet, D. Hannequin and E. Magnin, Amyloid-Related Imaging Abnormalities in $\mathrm{A} \beta \mathrm{PP}$ Duplication Carriers (4) 789-793

Chaunu, M.-P., V. Deramecourt, V. Buée-Scherrer, I.L. Ber, A. Brice, N. Ehrle, K.E. Hachimi, M. Pluot, C.-A. Maurage, S. Bakchine and L. Buée, Juvenile Frontotemporal Dementia with Parkinsonism Associated with Tau Mutation G389R (4) 769-776

Chen, H., see Ding, J. (3) 587-592

Chen, M., see Nguyen, H.T. (4) 835-848

Chen, N.-N., see Duan, D.-X. (4) 795-808

Chen, S., see Zhao, L. (2) 403-419

Cheng, J., see Cheng, X. (4) 823-834

Cheng, J., see Jantaratnotai, N. (2) 367-377

Cheng, X., Y. Zhou, W. Gu, J. Wu, A. Nie, J. Cheng, J. Zhou, W. Zhou and Y. Zhang, The Selective BACE1 Inhibitor VIa Reduces Amyloid- $\beta$ Production in Cell and Mouse Models of Alzheimer's Disease (4) 823-834

Cheng, X.-S., see Duan, D.-X. (4) 795-808

Cheng, Z., see Jin, C. (2) 429-437

Clodomiro, A., see Bernardi, L. (2) 285-289

Coelho, M., see Pires, C. (2) 335-342

Colao, R., see Bernardi, L. (2) 285-289

Conidi, M.E., see Bernardi, L. (2) 285-289

Connor, T.J., see Kelly, R.J. (1) 63-75

Cook, D.G., see Huber, B.R. (2) 309-323

Corrigan, B., see Ito, K. (1) 173-183

Cosentino, S., see Mez, J. (1) 137-146

Costello, D.A., see Kelly, R.J. (1) 63-75

Craigen, W.J., see Manczak, M. (4) 679-690

Cui, L., Y. Zhang, H. Cao, Y. Wang, T. Teng, G. Ma, Y. Li, K. Li and Y. Zhang, Ferulic Acid Inhibits the Transition of Amyloid- $\beta_{42}$ Monomers to Oligomers but Accelerates the Transition from Oligomers to Fibrils (1) 19-28

Curcio, S.A.M., see Bernardi, L. (2) 285-289

D’Addario, C. , see Di Francesco, A. (1) 3-8

D'Hooge, R., see Lo, A.C. (1) 109-125

D’Hooge, R., see Van der Jeugd, A. (4) 777-788
Dainese, E., see Di Francesco, A. (1) 3-8

Daschil, N., G.J. Obermair, B.E. Flucher, N. Stefanova, B. Hutter-Paier, M. Windisch, C. Humpel and J. Marksteiner, $\mathrm{Ca}_{\mathrm{v}} 1.2$ Calcium Channel Expression in Reactive Astrocytes is associated with the Formation of Amyloid- $\beta$ Plaques in an Alzheimer's Disease Mouse Model (2) 439-451

de Mendonça, A., see Pires, C. (2) 335-342

De Strooper, B., see Lo, A.C. (1) 109-125

del Carmen Alonso, A., see Beharry, C. (3) 539-550

del Carmen Alonso, A., see Gong, C.-X. (3) 465-466

Deng, Y., see Xiong, H. (3) 623-635

Denieffe, S., see Kelly, R.J. (1) 63-75

Deramecourt, V., see Chaunu, M.-P. (4) 769-776

Derisbourg, M., see Van der Jeugd, A. (4) 777-788

Descamps, O., P. Spilman, Q. Zhang, C.P. Libeu, K. Poksay, O. Gorostiza, J. Campagna, B. Jagodzinska, D.E. Bredesen and V. John, A $\beta$ PPSelective BACE Inhibitors (ASBI): Novel Class of Therapeutic Agents for Alzheimer's Disease (2) 343-355

Di Francesco, A., B. Arosio, C. Gussago, E. Dainese, D. Mari, C. D'Addario and M. Maccarrone, Involvement of 5-Lipoxygenase in Alzheimer's Disease: A Role for DNA Methylation (1) 3-8

Di Lorenzo, R., see Bernardi, L. (2) 285-289

Ding, J., Z. He, J. Ruan, Z. Ma, Y. Liu, C. Gong, K. Iqbal, S. Sun and H. Chen, Role of Ciliary Neurotrophic Factor in the Proliferation and Differentiation of Neural Stem Cells (3) 587-592

Dohmoto, C., see Noguchi-Shinohara, M. (4) 691-698

Duan, D.-X., G.-S. Chai, Z.-F. Ni, Y. Hu, Y. Luo, X.S. Cheng, N.-N. Chen, J.-Z. Wang and G.-P. Liu, Phosphorylation of Tau by Death-Associated Protein Kinase 1 Antagonizes the Kinase-Induced Cell Apoptosis (4) 795-808

Duff, K., see Yuan, A. (3) 579-586

Duque, G., see Annweiler, C. (1) 147-171

Dursun, E., see Gezen-Ak, D. (1) 185-195

Duyckaerts, C., see Pires, C. (2) 335-342

Ehrle, N., see Chaunu, M.-P. (4) 769-776

Emek-Savaş, D.D., see Yener, G.G. (4) 759-767

Emery, M.J., see Huber, B.R. (2) 309-323

Fadda, L., see Perri, R. (1) 99-107

Flory, M., see Iqbal, K. (3) 475-481

Flucher, B.E., see Daschil, N. (2) 439-451

Forte, E., see Krako, N. (4) 747-758

Fox, M., C. Berzuini and L.A. Knapp, Maternal Breastfeeding History and Alzheimer's Disease Risk (4) 809-821 
Frangipane, F., see Bernardi, L. (2) 285-289

Fratiglioni, L., see Handels, R.L.H. (2) 357-365

Frölich, L., see Morgen, K. (2) 389-401

Fu, Y., see Ward, S.M. (3) 593-602

Gallo, M., see Bernardi, L. (2) 285-289

Gao, Y., see Wu, N. (4) 699-712

García-García, E., see Hernández, F. (3) 507-513

Garthe, A., see Wiesmann, M. (1) 233-245

Gawinecka, J., M. Nowak, J. Carimalo, F. Cardone, A.R. Asif, W.M. Wemheuer, W.J. Schulz-Schaeffer, M. Pocchiari and I. Zerr, Subtype-Specific Synaptic Proteome Alterations in Sporadic CreutzfeldtJakob Disease (1) 51-61

Gellerfors, P., see Magnusson, K. (1) 29-40

Gertz, H.-J., see Morgen, K. (2) 389-401

Gezen-Ak, D., E. Dursun, H. Hanağası, B. Bilgiç, E. Lohman, Ö.S. Araz, İ.L. Atasoy, M. Alaylıoğlu, B. Önal, H. Gürvit and S. Yılmazer, BDNF, TNF $\alpha$, HSP90, CFH, and IL-10 Serum Levels in Patients with Early or Late Onset Alzheimer's Disease or Mild Cognitive Impairment (1) 185-195

Giuffrè, A., see Krako, N. (4) 747-758

Godoy, J.A., see Zolezzi, J.M. (4) 735-746

Gong, C., see Ding, J. (3) 587-592

Gong, C.-X., and A. del Carmen Alonso, Tau and Beyond for Alzheimer's Disease (3) 465-466

Gong, C.-X., see Qian, W. (3) 529-538

Gong, C.-X., see Yang, Y. (3) 637-648

Gordon, B.A., T. Blazey, T.L.S. Benzinger and D. Head, Effects of Aging and Alzheimer's Disease Along the Longitudinal Axis of the Hippocampus (1) $41-50$

Gorostiza, O., see Descamps, O. (2) 343-355

Götz, J., see Liu, C. (3) 483-494

Groblewska, M., see Mroczko, B. (2) 273-283

Grundke-Iqbal, I. (3) 467

Gu, W., see Cheng, X. (4) 823-834

Güntekin, B., see Yener, G.G. (4) 759-767

Gürvit, H., see Gezen-Ak, D. (1) 185-195

Gussago, C., see Di Francesco, A. (1) 3-8

Haapasalo, A., see Natunen, T. (1) 217-232

Hachimi, K.E., see Chaunu, M.-P. (4) 769-776

Hall, H., see Magnusson, K. (1) 29-40

Hallbeck, M., see Zheng, L. (4) 713-733

Hamdane, M., see Van der Jeugd, A. (4) 777-788

Hampel, H., see Morgen, K. (2) 389-401

Hampel, H., see Taverna, M. (4) 667-678

Han, F., see Hou, L. (1) 9-18

Hanağası, H., see Gezen-Ak, D. (1) 185-195
Handels, R.L.H., W. Xu, D. Rizzuto, B. Caracciolo, R. Wang, B. Winblad, F.R.J. Verhey, J.L. Severens, L. Fratiglioni, M.A. Joore and A. Wimo, Natural Progression Model of Cognition and Physical Functioning among People with Mild Cognitive Impairment and Alzheimer's Disease (2) 357-365

Hannequin, D., see Chamard, L. (4) 789-793

Hanson, R.J., see Smith, J.C. (1) 197-215

Hantke, N.C., see Smith, J.C. (1) 197-215

Hasselbalch, S.G., see Sorensen, K.C.N. (2) 379-387

Hattori, N., see Shiota, S. (2) 325-333

Hayashi, Y., see Nihonmatsu-Kikuchi, N. (3) 611-621

He, H.-J., see Lu, Y. (3) 551-563

He, R.-Q., see Lu, Y. (3) 551-563

He, Y.-G., see Lu, Y. (3) 551-563

He, Z., see Ding, J. (3) 587-592

Head, D., see Gordon, B.A. (1) 41-50

Heegaard, N.H.H., see Sorensen, K.C.N. (2) 379-387

Helisalmi, S., see Natunen, T. (1) 217-232

Hernández, F., E. García-García and J. Avila, Microtubule Depolymerization and Tau Phosphorylation Dedicated to Inge Grundke-Iqbal (3) 507-513

Heuser, I., see Morgen, K. (2) 389-401

Heydari, P., see Raven, E.P. (1) 127-136

Hiltunen, M., see Natunen, T. (1) 217-232

Himmelstein, D.S., see Ward, S.M. (3) 593-602

Holmetoft, U.B., see Sorensen, K.C.N. (2) 379-387

Holmquist, M., see Magnusson, K. (1) 29-40

Hou, L., H.-g. Lee, F. Han, J.M. Tedesco, G. Perry, M.A. Smith and M.G. Zagorski, Modification of Amyloid- $\beta_{1-42}$ Fibril Structure by Methionine-35 Oxidation (1) 9-18

Hu, N., see Sun, L. (2) 291-295

Hu, S., see Yang, Y. (3) 637-648

Hu, Y., see Duan, D.-X. (4) 795-808

Huber, B.R., J.S. Meabon, T.J. Martin, P.D. Mourad, R. Bennett, B.C. Kraemer, I. Cernak, E.C. Petrie, M.J. Emery, E.R. Swenson, C. Mayer, E. Mehic, E.R. Peskind and D.G. Cook, Blast Exposure Causes Early and Persistent Aberrant Phospho- and Cleaved-Tau Expression in a Murine Model of Mild Blast-Induced Traumatic Brain Injury (2) 309-323

Huey, E.D., see Mez, J. (1) 137-146

Hüll, M., see Morgen, K. (2) 389-401

Hultenby, K., see Zheng, L. (4) 713-733

Humpel, C., see Daschil, N. (2) 439-451

Hutter-Paier, B., see Daschil, N. (2) 439-451

Ikeda, Y., see Noguchi-Shinohara, M. (4) 691-698

Inestrosa, N.C., see Zolezzi, J.M. (4) 735-746 
Iqbal, K., see Ding, J. (3) 587-592

Iqbal, K., M. Flory and H. Soininen, Clinical Symptoms and Symptom Signatures of Alzheimer's Disease Subgroups (3) 475-481

Iqbal, K., S. Bolognin, X. Wang, G. Basurto-Islas, J. Blanchard and Y.C. Tung, Animal Models of the Sporadic Form of Alzheimer's Disease: Focus on the Disease and Not Just the Lesions (3) 469-474

Iqbal, K., see Qian, W. (3) 529-538

Iscru, E., see Lo, A.C. (1) 109-125

Ito, K., B. Corrigan, K. Romero, R. Anziano, J. Neville, D. Stephenson and R. Lalonde, Understanding Placebo Responses in Alzheimer's Disease Clinical Trials from the Literature Meta-Data and CAMD Database (1) 173-183

Iwasa, K., see Noguchi-Shinohara, M. (4) 691-698

Jadhav, S., see Levarska, L. (3) 569-577

Jagodzinska, B., see Descamps, O. (2) 343-355

Jahn, H., see Morgen, K. (2) 389-401

Jansen, D., see Wiesmann, M. (1) 233-245

Jantaratnotai, N., A. Ling, J. Cheng, C. Schwab, P.L. McGeer and J.G. McLarnon, Upregulation and Expression Patterns of the Angiogenic Transcription Factor Ets-1 in Alzheimer's Disease Brain (2) 367-377

Jeong, Y., see Kim, J. (4) 857868

Jessen, F., see Morgen, K. (2) 389-401

Jiménez, J.M., see Morales, I. (4) 849-856

Jin, C., X. Liu, F. Zhang, Y. Wu, J. Yuan, J. Zhu, F. Zhang, G. Wang and Z. Cheng, An Updated MetaAnalysis of the Association between SORL1 Variants and the Risk for Sporadic Alzheimer's Disease (2) 429-437

Jin, N., see Qian, W. (3) 529-538

Jin, X., see Qian, W. (3) 529-538

John, V., see Descamps, O. (2) 343-355

Jones, R.S., see Kelly, R.J. (1) 63-75

Joore, M.A., see Handels, R.L.H. (2) 357-365

Kazmerova, Z., N. Zilka, M. Cente, P. Neradil, M. Zilkova and M. Novak, Can We Teach Old Dogs New Tricks? Neuroprotective Cell Therapy in Alzheimer's and Parkinson's Disease (2) 251272

Kelly, R.J., A.M. Minogue, A. Lyons, R.S. Jones, T.C. Browne, D.A. Costello, S. Denieffe, C. O’Sullivan, T.J. Connor and M.A. Lynch, Glial Activation in

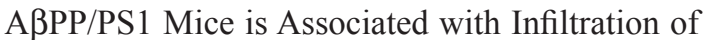
IFN $\gamma$-Producing Cells (1) 63-75

Kiliaan, A.J., see Wiesmann, M. (1) 233-245
Kim, E.-M., see O’Hare, E. (1) 77-88

Kim, J., and Y. Jeong, Augmentation of Sensory-Evoked Hemodynamic Response in an Early Alzheimer's Disease Mouse Model (4) 857-868

Knapp, L.A., see Fox, M. (4) 809-821

Kölsch, H., see Morgen, K. (2) 389-401

Komai, K., see Noguchi-Shinohara, M. (4) 691-698

Kornhuber, J., see Morgen, K. (2) 389-401

Kowall, N., see Qiu, W.Q. (2) 421-428

Kraemer, B.C., see Huber, B.R. (2) 309-323

Krako, N., M.C. Magnifico, M. Arese, G. Meli, E. Forte, A. Lecci, A. Manca, A. Giuffrè, D. Mastronicola, P. Sarti and A. Cattaneo, Characterization of Mitochondrial Dysfunction in the 7PA2 Cell Model of Alzheimer's Disease (4) 747-758

Kumar, A., see Yuan, A. (3) 579-586

Kurkinen, K.M.A., see Natunen, T. (1) 217-232

Kurt, P., see Yener, G.G. (4) 759-767

Lalonde, R., see Ito, K. (1) 173-183

Lancia, J.K., see Ward, S.M. (3) 593-602

Lannfelt, L., see Magnusson, K. (1) 29-40

Larner, A.J., Presenilin-1 Mutations in Alzheimer's Disease: An Update on Genotype-Phenotype Relationships (4) 653-659

Lecci, A., see Krako, N. (4) 747-758

Lee, H.-g., see Hou, L. (1) 9-18

Levarska, L., N. Zilka, S. Jadhav, P. Neradil and M. Novak, Of Rodents and Men: The Mysterious Interneuronal Pilgrimage of Misfolded Protein Tau in Alzheimer's Disease Dedicated to the memory of Prof. Inge-Grundke Iqbal: A leader in $A D$ research (3) 569-577

Li, H., see Qiu, W.Q. (2) 421-428

Li, K., see Cui, L. (1) 19-28

Li, Y., see Cui, L. (1) 19-28

Libeu, C.P., see Descamps, O. (2) 343-355

Lichtenthaler, S.F., see Taverna, M. (4) 667-678

Ling, A., see Jantaratnotai, N. (2) 367-377

Liu, C., and J. Götz, How it all Started: Tau and Protein Phosphatase 2A Dedicated to Inge Grundke-Iqbal (3) 483-494

Liu, D., see Wu, Y.-Y. (3) 515-527

Liu, F., see Qian, W. (3) 529-538

Liu, G.-P., see Duan, D.-X. (4) 795-808

Liu, X., see Jin, C. (2) 429-437

Liu, X.-H., see Wu, Y.-Y. (3) 515-527

Liu, Y., see Ding, J. (3) 587-592

Liu, Y., see Lu, Y. (3) 551-563

Llewellyn, D.J., see Annweiler, C. (1) 147-171

Lo, A.C., see Van der Jeugd, A. (4) 777-788 
Lo, A.C., E. Iscru, D. Blum, I. Tesseur, Z. CallaertsVegh, L. Buée, B. De Strooper, D. Balschun and R. D'Hooge, Amyloid and Tau Neuropathology Differentially Affect Prefrontal Synaptic Plasticity and Cognitive Performance in Mouse Models of Alzheimer's Disease (1) 109-125

Lohman, E., see Gezen-Ak, D. (1) 185-195

Lu, J., see Lu, Y. (3) 551-563

Lu, P.H., see Raven, E.P. (1) 127-136

Lu, Y., H.-J. He, J. Zhou, J.-Y. Miao, J. Lu, Y.G. He, R. Pan, Y. Wei, Y. Liu and R.-Q. He, Hyperphosphorylation Results in Tau Dysfunction in DNA Folding and Protection (3) 551-563

Luckhaus, C., see Morgen, K. (2) 389-401

Luo, Y., see Duan, D.-X. (4) 795-808

Lynch, M.A., see Kelly, R.J. (1) 63-75

Lyons, A., see Kelly, R.J. (1) 63-75

Lyons, J.-A., see Smith, J.C. (1) 197-215

Ma, D., see Yang, Y. (3) 637-648

Ma, G., see Cui, L. (1) 19-28

Ma, Z., see Ding, J. (3) 587-592

Maccarrone, M., see Di Francesco, A. (1) 3-8

Maccioni, R.B., see Morales, I. (4) 849-856

Magnifico, M.C., see Krako, N. (4) 747-758

Magnin, E., see Chamard, L. (4) 789-793

Magnusson, K., D. Sehlin, S. Syvänen, M.M. Svedberg, O. Philipson, L. Söderberg, K. Tegerstedt, M. Holmquist, P. Gellerfors, V. Tolmachev, G. Antoni, L. Lannfelt, H. Hall and L.N.G. Nilsson, Specific Uptake of an Amyloid- $\beta$ Protofibril-Binding Antibody-Tracer in A $\beta P P$ Transgenic Mouse Brain (1) 29-40

Maier, W., see Morgen, K. (2) 389-401

Mäkinen, P., see Natunen, T. (1) 217-232

Maletta, R., see Bernardi, L. (2) 285-289

Manca, A., see Krako, N. (4) 747-758

Mancilla, M., see Morales, I. (4) 849-856

Manczak, M., T. Sheiko, W.J. Craigen and P.H. Reddy, Reduced VDAC1 Protects Against Alzheimer's Disease, Mitochondria, and Synaptic Deficiencies (4) 679-690

Mao, Z., see Zhao, L. (2) 403-419

Marcusson, J., see Zheng, L. (4) 713-733

Mari, D., see Di Francesco, A. (1) 3-8

Mariani, S., see Bucossi, S. (2) 453-459

Markov, O., see Nguyen, H.T. (4) 835-848

Marksteiner, J., see Daschil, N. (2) 439-451

Martin, T.J., see Huber, B.R. (2) 309-323

Martins, M., see Pires, C. (2) 335-342

Mastronicola, D., see Krako, N. (4) 747-758
Matsumoto, S.-e., see Shiota, S. (2) 325-333

Maurage, C.-A., see Chaunu, M.-P. (4) 769-776

Mayer, C., see Huber, B.R. (2) 309-323

Mayeux, R., see Mez, J. (1) 137-146

McGeer, P.L., see Jantaratnotai, N. (2) 367-377

McLarnon, J.G., see Jantaratnotai, N. (2) 367-377

Meabon, J.S., see Huber, B.R. (2) 309-323

Mehic, E., see Huber, B.R. (2) 309-323

Meli, G., see Krako, N. (4) 747-758

Meyer-Lindenberg, A., see Morgen, K. (2) 389-401

Mez, J., S. Cosentino, A.M. Brickman, E.D. Huey and R. Mayeux, Different Demographic, Genetic, and Longitudinal Traits in Language versus Memory Alzheimer's Subgroups (1) 137-146

Miao, J.-Y., see Lu, Y. (3) 551-563

Miltenberger-Miltenyi, G., see Pires, C. (2) 335-342

Minogue, A.M., see Kelly, R.J. (1) 63-75

Mirabelli, M., see Bernardi, L. (2) 285-289

Mochizuki, H., see Shiota, S. (2) 325-333

Montero-Odasso, M., see Annweiler, C. (1) 147-171

Morales, I., J.M. Jiménez, M. Mancilla and R.B. Maccioni, Tau Oligomers and Fibrils Induce Activation of Microglial Cells (4) 849-856

Morgen, K., L. Frölich, H. Tost, M.M. Plichta, H. Kölsch, F. Rakebrandt, O. Rienhoff, F. Jessen, O. Peters, H. Jahn, C. Luckhaus, M. Hüll, H.-J. Gertz, J. Schröder, H. Hampel, S.J. Teipel, J. Pantel, I. Heuser, J. Wiltfang, E. Rüther, J. Kornhuber, W. Maier and A. Meyer-Lindenberg, APOEDependent Phenotypes in Subjects with Mild Cognitive Impairment Converting to Alzheimer's Disease (2) 389-401

Mourad, P.D., see Huber, B.R. (2) 309-323

Mroczko, B., M. Groblewska and M. Barcikowska, The Role of Matrix Metalloproteinases and Tissue Inhibitors of Metalloproteinases in the Pathophysiology of Neurodegeneration: A Literature Study (2) 273-283

Mullin, K., see Natunen, T. (1) 217-232

Mwamburi, M., see Qiu, W.Q. (2) 421-428

Nakamura, H., see Noguchi-Shinohara, M. (4) 691-698 Natunen, T., A.R. Parrado, S. Helisalmi, J.-P. Pursiheimo, T. Sarajärvi, P. Mäkinen, K.M.A. Kurkinen, K. Mullin, I. Alafuzoff, A. Haapasalo, L. Bertram, H. Soininen, R.E. Tanzi and M. Hiltunen, Elucidation of the BACE1 Regulating Factor GGA3 in Alzheimer's Disease (1) 217-232 Neradil, P., see Kazmerova, Z. (2) 251-272

Neradil, P., see Levarska, L. (3) 569-577

Neville, J., see Ito, K. (1) 173-183 
Nguyen, H.T., D.R. Sawmiller, O. Markov and M. Chen, Elevated $\left[\mathrm{Ca}^{2+}\right]_{\mathrm{i}}$ Levels Occur with Decreased Calpain Activity in Aged Fibroblasts and Their Reversal by Energy-Rich Compounds: New Paradigm for Alzheimer's Disease Prevention (4) 835-848

Ni, Z.-F., see Duan, D.-X. (4) 795-808

Nie, A., see Cheng, X. (4) 823-834

Nie, S.-D., see Wang, S. (3) 495-505

Nielson, K.A., see Smith, J.C. (1) 197-215

Nihonmatsu-Kikuchi, N., Y. Hayashi, X.-j. Yu and Y. Tatebayashi, Depression and Alzheimer's Disease: Novel Postmortem Brain Studies Reveal a Possible Common Mechanism (3) 611-621

Nilsson, L.N.G., see Magnusson, K. (1) 29-40

Nixon, R.A., see Yuan, A. (3) 579-586

Noguchi-Shinohara, M., S. Yuki, C. Dohmoto, Y. Ikeda, M. Samuraki, K. Iwasa, M. Yokogawa, K. Asai, K. Komai, H. Nakamura and M. Yamada, Differences in the Prevalence of Dementia and Mild Cognitive Impairment and Cognitive Functions between Early and Delayed Responders in a CommunityBased Study of the Elderly (4) 691-698

Novak, M., see Kazmerova, Z. (2) 251-272

Novak, M., see Levarska, L. (3) 569-577

Nowak, M., see Gawinecka, J. (1) 51-61

Nurwidya, F., see Shiota, S. (2) 325-333

O'Hare, E., T. Ardis, D. Page, D.I.C. Scopes and E.-M. Kim, A $\beta$ PP-Overexpressing Transgenic Rat Model of Alzheimer's Disease Utilizing the Tg2576 Mouse Protocol (1) 77-88

O’Sullivan, C., see Kelly, R.J. (1) 63-75

Obermair, G.J., see Daschil, N. (2) 439-451

Önal, B., see Gezen-Ak, D. (1) 185-195

Ordenes, D., see Zolezzi, J.M. (4) 735-746

Page, D., see O'Hare, E. (1) 77-88

Palmer, R.F., see Royall, D.R. (1) 89-97

Pan, R., see Lu, Y. (3) 551-563

Pantel, J., see Morgen, K. (2) 389-401

Parrado, A.R., see Natunen, T. (1) 217-232

Patterson, K.R., see Ward, S.M. (3) 593-602

Pei, J.-J., see Wang, S. (3) 495-505

Perri, R., L. Fadda, C. Caltagirone and G.A. Carlesimo, Word List and Story Recall Elicit Different Patterns of Memory Deficit in Patients with Alzheimer's Disease, Frontotemporal Dementia, Subcortical Ischemic Vascular Disease, and Lewy Body Dementia (1) 99-107

Perry, G., see Hou, L. (1) 9-18
Peskind, E.R., see Huber, B.R. (2) 309-323

Peters, O., see Morgen, K. (2) 389-401

Petrie, E.C., see Huber, B.R. (2) 309-323

Philipson, O., see Magnusson, K. (1) 29-40

Phillips, L., see Qiu, W.Q. (2) 421-428

Pijoff, A., see Chamard, L. (4) 789-793

Pimentel, J., see Pires, C. (2) 335-342

Pires, C., M. Coelho, A. Valadas, C. Barroso, J. Pimentel, M. Martins, C. Duyckaerts, A. de Mendonça, A. Verdelho and G. MiltenbergerMiltenyi, Phenotypic Variability of Familial and Sporadic Progranulin p.Gln257Profs*27 Mutation (2) $335-342$

Plichta, M.M., see Morgen, K. (2) 389-401

Pluot, M., see Chaunu, M.-P. (4) 769-776

Pocchiari, M., see Gawinecka, J. (1) 51-61

Poksay, K., see Descamps, O. (2) 343-355

Polimanti, R., see Bucossi, S. (2) 453-459

Popova, S.N., and I. Alafuzoff, Distribution of SLC10A4, a Synaptic Vesicle Protein in the Human Brain, and the Association of this Protein with Alzheimer's Disease-Related Neuronal Degeneration (3) 603-610

Puccio, G., see Bernardi, L. (2) 285-289

Pursiheimo, J.-P., see Natunen, T. (1) 217-232

Qian, W., N. Jin, J. Shi, X. Yin, X. Jin, S. Wang, M. Cao, K. Iqbal, C.-X. Gong and F. Liu, Dualspecificity Tyrosine Phosphorylation-regulated Kinase 1A (Dyrk1A) Enhances Tau Expression (3) $529-538$

Qiao, L., see Qiu, W.Q. (2) 421-428

Qiu, W.Q., M. Mwamburi, L.M. Besser, H. Zhu, H. Li, M. Wallack, L. Phillips, L. Qiao, A.E. Budson, R. Stern and N. Kowall, Angiotensin Converting Enzyme Inhibitors and the Reduced Risk of Alzheimer's Disease in the Absence of Apolipoprotein E4 Allele (2) 421-428

Rakebrandt, F., see Morgen, K. (2) 389-401

Rao, X., see Wu, N. (4) 699-712

Raven, E.P., P.H. Lu, T.A. Tishler, P. Heydari and G. Bartzokis, Increased Iron Levels and Decreased Tissue Integrity in Hippocampus of Alzheimer's Disease Detected in vivo with Magnetic Resonance Imaging (1) 127-136

Reddy, P.H., see Manczak, M. (4) 679-690

Richard-Devantoy, S., see Annweiler, C. (1) 147-171

Rienhoff, O., see Morgen, K. (2) 389-401

Ríos, J.A., see Zolezzi, J.M. (4) 735-746

Rizzuto, D., see Handels, R.L.H. (2) 357-365 
Robinson, R.A.S., Z. Cao and C. Williams, Oxidative Stress in CD90+ T-cells of A $\beta$ PP/PS-1 Transgenic Mice (4) 661-666

Romero, K., see Ito, K. (1) 173-183

Royall, D.R., R.F. Palmer for the Texas Alzheimer's Research and Care Consortium, Validation of a Latent Construct for Dementia Case-Finding in Mexican-Americans (1) 89-97

Ruan, J., see Ding, J. (3) 587-592

Rujescu, D., see Taverna, M. (4) 667-678

Rüther, E., see Morgen, K. (2) 389-401

Sabbagh, M.N., Larry Sparks: Innovator and Iconoclast (1) 1

Samuraki, M., see Noguchi-Shinohara, M. (4) 691-698

Sarajärvi, T., see Natunen, T. (1) 217-232

Sarti, P., see Krako, N. (4) 747-758

Sasaki, T., see Yuan, A. (3) 579-586

Sawmiller, D.R., see Nguyen, H.T. (4) 835-848

Schneider, L.S., see Zhao, L. (2) 403-419

Schröder, J., see Morgen, K. (2) 389-401

Schulz-Schaeffer, W.J., see Gawinecka, J. (1) 51-61

Schwab, C., see Jantaratnotai, N. (2) 367-377

Scopes, D.I.C., see O'Hare, E. (1) 77-88

Scrascia, F., see Bucossi, S. (2) 453-459

Sehlin, D., see Magnusson, K. (1) 29-40

Severens, J.L., see Handels, R.L.H. (2) 357-365

Shao, S., see Yang, Y. (3) 637-648

Sheiko, T., see Manczak, M. (4) 679-690

Shen, H., see Xiong, H. (3) 623-635

Shi, J., see Qian, W. (3) 529-538

Shiota, S., H. Takekawa, S.-e. Matsumoto, K. Takeda, F. Nurwidya, Y. Yoshioka, F. Takahashi, N. Hattori, T. Tabira, H. Mochizuki and K. Takahashi, Chronic Intermittent Hypoxia/Reoxygenation Facilitate Amyloid- $\beta$ Generation in Mice (2) 325-333

Silva-Alvarez, C., see Zolezzi, J.M. (4) 735-746

Simonsen, A.H., see Sorensen, K.C.N. (2) 379-387

Siotto, M., see Bucossi, S. (2) 453-459

Smirne, N., see Bernardi, L. (2) 285-289

Smith, J.C., K.A. Nielson, P. Antuono, J.-A. Lyons, R.J. Hanson, A.M. Butts, N.C. Hantke and M.D. Verber, Semantic Memory Functional MRI and Cognitive Function after Exercise Intervention in Mild Cognitive Impairment (1) 197-215

Smith, M.A., see Hou, L. (1) 9-18

Söderberg, L., see Magnusson, K. (1) 29-40

Soininen, H., see Iqbal, K. (3) 475-481

Soininen, H., see Natunen, T. (1) 217-232

Song, J., see Xiong, H. (3) 623-635

Sorensen, K.C.N., A.H. Simonsen, U.B. Holmetoft, S.G. Hasselbalch and N.H.H. Heegaard,
Neprilysin-Like Activity Correlates with CSF-Tau and Phospho-Tau in Patients with Alzheimer's Disease (2) 379-387

Spilman, P., see Descamps, O. (2) 343-355

Squitti, R., see Bucossi, S. (2) 453-459

Stefanova, N., see Daschil, N. (2) 439-451

Stephenson, D., see Ito, K. (1) 173-183

Stern, R., see Qiu, W.Q. (2) 421-428

Straub, T., see Taverna, M. (4) 667-678

Sun, L., M.-S. Tan, N. Hu, J.-T. Yu and L. Tan, Exploring the Value of Plasma BIN1 as a Potential Biomarker for Alzheimer's Disease (2) 291-295

Sun, S., see Ding, J. (3) 587-592

Svedberg, M.M., see Magnusson, K. (1) 29-40

Swenson, E.R., see Huber, B.R. (2) 309-323

Syvänen, S., see Magnusson, K. (1) 29-40

Tabira, T., see Shiota, S. (2) 325-333

Takahashi, F., see Shiota, S. (2) 325-333

Takahashi, K., see Shiota, S. (2) 325-333

Takashima, A., Tauopathies and Tau Oligomers (3) 565-568

Takeda, K., see Shiota, S. (2) 325-333

Takekawa, H., see Shiota, S. (2) 325-333

Tan, L., see Sun, L. (2) 291-295

Tan, L., see Wu, Y.-Y. (3) 515-527

Tan, M.-S., see Sun, L. (2) 291-295

Tanzi, R.E. , see Natunen, T. (1) 217-232

Tatebayashi, Y., see Nihonmatsu-Kikuchi, N. (3) 611621

Taverna, M., T. Straub, H. Hampel, D. Rujescu and S.F. Lichtenthaler, A New Sandwich Immunoassay for Detection of the $\alpha$-Secretase Cleaved, Soluble Amyloid- $\beta$ Protein Precursor in Cerebrospinal Fluid and Serum (4) 667-678

Tedesco, J.M., see Hou, L. (1) 9-18

Tegerstedt, K., see Magnusson, K. (1) 29-40

Teipel, S.J., see Morgen, K. (2) 389-401

Teng, T., see Cui, L. (1) 19-28

Terman, A., see Zheng, L. (4) 713-733

Tesseur, I., see Lo, A.C. (1) 109-125

Tishler, T.A., see Raven, E.P. (1) 127-136

Tolmachev, V., see Magnusson, K. (1) 29-40

Tost, H., see Morgen, K. (2) 389-401

Trotta, L., see Bucossi, S. (2) 453-459

Tung, Y.C., see Iqbal, K. (3) 469-474

Ursini, F., see Bucossi, S. (2) 453-459

Valadas, A., see Pires, C. (2) 335-342

Van der Jeugd, A., B. Vermaercke, M. Derisbourg, A.C. Lo, M. Hamdane, D. Blum, L. Buée and 
R. D’Hooge, Progressive Age-Related Cognitive Decline in Tau Mice (4) 777-788

Vasso, F., see Bernardi, L. (2) 285-289

Ventriglia, M., see Bucossi, S. (2) 453-459

Verber, M.D., see Smith, J.C. (1) 197-215

Verdelho, A., see Pires, C. (2) 335-342

Verhey, F.R.J., see Handels, R.L.H. (2) 357-365

Vermaercke, B., see Van der Jeugd, A. (4) 777-788

Vernieri, F., see Bucossi, S. (2) 453-459

Viennet, G., see Chamard, L. (4) 789-793

Wallack, M., see Qiu, W.Q. (2) 421-428

Wallon, D., see Chamard, L. (4) 789-793

Wang, G., see Jin, C. (2) 429-437

Wang, J., see Wu, N. (4) 699-712

Wang, J., see Xiong, H. (3) 623-635

Wang, J.-Z., see Duan, D.-X. (4) 795-808

Wang, J.-Z., see Wu, Y.-Y. (3) 515-527

Wang, Q., see Wu, Y.-Y. (3) 515-527

Wang, R., see Handels, R.L.H. (2) 357-365

Wang, S., J. Wu, S.-D. Nie, E. Bereczki and J.-J. Pei, Dysregulated mTOR-Dependent Signaling in Neurodegeneration or Carcinogenesis: Implication for Alzheimer's Disease and Brain Tumors (3) 495-505

Wang, S., see Qian, W. (3) 529-538

Wang, X., see Iqbal, K. (3) 469-474

Wang, X., see Wu, Y.-Y. (3) 515-527

Wang, Y., see Cui, L. (1) 19-28

Ward, S.M., D.S. Himmelstein, J.K. Lancia, Y. Fu, K.R. Patterson and L.I. Binder, TOC1: Characterization of a Selective Oligomeric Tau Antibody (3) 593602

Wei, Y., see Lu, Y. (3) 551-563

Wemheuer, W.M., see Gawinecka, J. (1) 51-61

Wiesmann, M., D. Jansen, V. Zerbi, L.M. Broersen, A. Garthe and A.J. Kiliaan, Improved Spatial Learning Strategy and Memory in Aged Alzheimer A $\beta P P s w e / P S 1 d E 9$ Mice on a Multi-Nutrient Diet (1) 233-245

Williams, C., see Robinson, R.A.S. (4) 661-666

Wiltfang, J., see Morgen, K. (2) 389-401

Wimo, A., see Handels, R.L.H. (2) 357-365

Winblad, B., see Handels, R.L.H. (2) 357-365

Windisch, M., see Daschil, N. (2) 439-451

Wittnam, J.L., see Yang, H. (2) 297-308

Wu, J., see Cheng, X. (4) 823-834

Wu, J., see Wang, S. (3) 495-505

Wu, N., X. Rao, Y. Gao, J. Wang and F. Xu, Amyloid- $\beta$ Deposition and Olfactory Dysfunction in an Alzheimer's Disease Model (4) 699-712
Wu, Y., see Jin, C. (2) 429-437

Wu, Y.-Y., X. Wang, L. Tan, D. Liu, X.-H. Liu, Q. Wang, J.-Z. Wang and L.-Q. Zhu, Lithium Attenuates Scopolamine-Induced Memory Deficits with Inhibition of GSK-3 $\beta$ and Preservation of Postsynaptic Components (3) 515-527

Xiong, H., C. Zheng, J. Wang, J. Song, G. Zhao, H. Shen and Y. Deng, The Neuroprotection of Liraglutide on Alzheimer-Like Learning and Memory Impairment by Modulating the Hyperphosphorylation of Tau and Neurofilament Proteins and Insulin Signaling Pathways in Mice (3) 623-635

Xu, F., see Wu, N. (4) 699-712

$\mathrm{Xu}$, W., see Handels, R.L.H. (2) 357-365

Yamada, M., see Noguchi-Shinohara, M. (4) 691-698

Yang, H., J.L. Wittnam, R.A. Zubarev and T.A. Bayer, Shotgun Brain Proteomics Reveals Early Molecular Signature in Presymptomatic Mouse Model of Alzheimer's Disease (2) 297-308

Yang, Y., J. Zhang, D. Ma, M. Zhang, S. Hu, S. Shao and C.-X. Gong, Subcutaneous Administration of Liraglutide Ameliorates Alzheimer-Associated Tau Hyperphosphorylation in Rats with Type 2 Diabetes (3) 637-648

Yener, G.G., P. Kurt, D.D. Emek-Savaş, B. Güntekin and E. Başar, Reduced Visual Event-Related Delta Oscillatory Responses in Amnestic Mild Cognitive Impairment (4) 759-767

Y1lmazer, S., see Gezen-Ak, D. (1) 185-195

Yin, X., see Qian, W. (3) 529-538

Yokogawa, M., see Noguchi-Shinohara, M. (4) 691-698

Yoshioka, Y., see Shiota, S. (2) 325-333

Yu, J.-T., see Sun, L. (2) 291-295

Yu, X.-j., see Nihonmatsu-Kikuchi, N. (3) 611-621

Yuan, A., A. Kumar, T. Sasaki, K. Duff and R.A. Nixon, Global Axonal Transport Rates are Unaltered in Htau Mice in vivo (3) 579-586

Yuan, J., see Jin, C. (2) 429-437

Yuki, S., see Noguchi-Shinohara, M. (4) 691-698

Zagorski, M.G., see Hou, L. (1) 9-18

Zerbi, V., see Wiesmann, M. (1) 233-245

Zerr, I., see Gawinecka, J. (1) 51-61

Zhang, F., see Jin, C. (2) 429-437

Zhang, F., see Jin, C. (2) 429-437

Zhang, J., see Yang, Y. (3) 637-648

Zhang, M., see Yang, Y. (3) 637-648

Zhang, Q., see Descamps, O. (2) 343-355 
Zhang, Y., see Cheng, X. (4) 823-834

Zhang, Y., see Cui, L. (1) 19-28

Zhang, Y., see Cui, L. (1) 19-28

Zhao, G., see Xiong, H. (3) 623-635

Zhao, L., Z. Mao, S. Chen, L.S. Schneider and R.D. Brinton, Early Intervention with an Estrogen Receptor $\beta$-Selective Phytoestrogenic Formulation Prolongs Survival, Improves Spatial Recognition Memory, and Slows Progression of Amyloid Pathology in a Female Mouse Model of Alzheimer's Disease (2) 403-419

Zheng, C., see Xiong, H. (3) 623-635

Zheng, L., J. Calvo-Garrido, M. Hallbeck, K. Hultenby, J. Marcusson, A. Cedazo-Minguez and A. Terman, Intracellular Localization of Amyloid- $\beta$ Peptide in SH-SY5Y Neuroblastoma Cells (4) 713-733

Zhou, J., see Cheng, X. (4) 823-834
Zhou, J., see Lu, Y. (3) 551-563

Zhou, W., see Cheng, X. (4) 823-834

Zhou, Y., see Cheng, X. (4) 823-834

Zhu, H., see Qiu, W.Q. (2) 421-428

Zhu, J., see Jin, C. (2) 429-437

Zhu, L.-Q., see Wu, Y.-Y. (3) 515-527

Zilka, N., see Kazmerova, Z. (2) 251-272

Zilka, N., see Levarska, L. (3) 569-577

Zilkova, M., see Kazmerova, Z. (2) 251-272

Zolezzi, J.M., F.J. Carvajal, J.A. Ríos, D. Ordenes, C. Silva-Alvarez, J.A. Godoy and N.C. Inestrosa, Tetrahydrohyperforin Induces Mitochondrial Dynamics and Prevents Mitochondrial $\mathrm{Ca}^{2+}$ Overload after $\mathrm{A} \beta$ and $\mathrm{A} \beta$-AChE Complex Challenge in Rat Hippocampal Neurons (4) 735746

Zubarev, R.A., see Yang, H. (2) 297-308 